\title{
ON OBSTRUCTIONS
}

OF THE

\section{BRANCHES OF THE PULMONARY ARTERY.}

By JAMES PAGET, F.R.C.S.,

LECTURER ON PHYSIOLOGY AND WARDEN OF THE COLLEGIATE ESTABLISHMENT AT ST. BARTHOLOMEW'S HOSPITAL.

READ APRIL 9TH, 1844.

The obstructions of which it is proposed to treat, are those which are produced by the coagulation of the blood during life in the branches of the pulmonary artery. They are not of rare occurrence, and are always important, for they are sometimes the sole or chief cause of death, and must in all cases seriously affect the progress of the diseases with which they are associated. Yet obstructions of this kind have hitherto scarcely attracted attention. In the best works on Morbid Anatomy and on Diseases of the Chest, they are either not alluded to, or are vaguely and incidentally mentioned; and in the only paper* which I can find expressly treating of them, there are but four cases related, and these, though

* “ Recherches et Observations sur la Coagulation du Sang dans l'Artère Pulmonaire et ses effets, par M. C. Baron." Archives Générales de Médecine, Ser. III. T. II. p. l. Paris, 1838. 
carefully detailed, are unconnected, and give a very imperfect account of the nature of the disease, and of the circumstances in which it occurs. I trust, therefore, that the following observations will be acceptable to the Society.

From the arrangement of the pulmonary arteries, between which there is no anastomosis, except in their capillaries and smallest branches, it results that whenever the flow of blood through the capillaries of any part of a lung is prevented, there must also be a stagnation of the blood in all the branches from which those capillaries are derived; and in these circumstances, the blood coagulates in the vessels, and passes through various changes.

Now, these conditions are present in several diseases :-First, in pulmonary apoplexy, especially in that form of it in which the blood collects in a defined and compact dark mass. At a meeting of the Society two years ago, I stated, what more recent examinations have confirmed, namely, that in all, or in the great majority of these cases, the branches of the pulmonary artery leading to the seat of effusion are blocked up by coagula, which present the distinguishing characters of those formed long previous to death. In most cases, (perhaps in all those which depend only on disease of the heart,) the coagula are found in those branches alone in which the circulation is obstructed, but in some instances they extend further, the apoplectic effusion being inconsiderable in comparison with the size and num- 
ber of the branches which are filled by coagula. These cases, examples of which will be described, make it highly probable that sometimes the formation of coagula in the pulmonary arteries, when found combined with pulmonary apoplexy, has been, not consequent on the effusion of blood, but coincident with it, or has, in some measure, preceded it.

Secondly.-The capillary circulation is usually obstructed, or much hindered, in the advanced stages of pneumonia, and the arrested blood coagulates in those branches which correspond to the inflamed and consolidated portions of lung. So far as I have seen, the coagula in these cases are not sufficiently large to fill the vessels; circulation enough, therefore, may go on to prevent gangrene; but still, such permanent, though imperfect obstacles to the passage of blood must materially impede and endanger the recovery of the diseased part, and no doubt the formation of these clots often precedes the complete obliteration of the pulmonary arteries, when, after partial recovery from pneumonia, the diseased portion of the lung has healed and contracted. Sometimes, indeed, coagula thus formed in pneumonia are fatal. They were so in one of the cases mentioned by M. Baron. A patient of M. Louis' was suddenly seized with symptoms of asphyxia during convalescence from pneumonia : he died in five or six hours, and "nothing could be found to explain his death, except some soft non. 
adherent clots, which obstructed the cavity of the pulmonary artery."**

Thirdly.-When the matter of medullary cancer or of softened scirrhus passes into the blood, and, circulating with it, is stopped in the lungs, the branches of the pulmonary artery may be to a great extent filled by it and by coagulated blood or fibrine mixed with it. In the following case there is a good example of this fact.

\section{CASE I.}

A woman of drunken habits, 45 years old, had been in St. Bartholomew's Hospital with all the symptoms and appearance of one suffering from cirrhosis. She could assign no distinct date to the commencement of her disease, and died very much emaciated, jaundiced, and with considerable ascites and anasarca of the lower extremities. On the examination of the body thirteen hours after death, I found (to mention only those parts which are of present interest) the liver large, hard, yellow and dry, and adherent to a contracted great omentum. It contained several medullary tumours composed of very soft tissue, out of which could be pressed a large quantity of pulpy matter, of golden or king's-yellow colour, mixed with various shades of green and brown. A quantity of the same matter, but more nearly fluid, seemed loosely effused in the substance of the liver, and some more of it

* L. c. p. 17 . 
was contained in the large hepatic blood-vessels. The lungs were nearly free from adhesions. In each of them, irregularly scattered through every part, there were nearly a hundred small masses of medullary matter of the same bright yellow colour as those in the liver. They did not form any tissue, but appeared loosely effused, so that after any one of them was cut through, each part of it could be wiped away without injuring the structure of the lung. A similar yellow substance, but apparently thinner and more nearly fluid, filled a number of the small branches of the pulmonary artery, in such a manner that, on a section of the lung, fine yellow ramified lines were seen, as if the arteries had been injected with chromate of lead and size, or some thick material. There could be no doubt that the cancerous substance had been conveyed with the blood from the liver to the lungs, where, being arrested and obstructing the minute vessels, it had permitted fresh substance with blood to accumulate behind it. There is a preparation from another case of the same kind in the Museum of St. Bartholomew's Hospital ;* but the characters are less marked, because the cancerous matter is uncoloured. I have notes of a third similar case ; and in another preparation in the same collection, $\dagger$ the trunk of the left pulmonary artery is filled by a large firm mass, apparently composed of fibrine, mixed with cancerous

* Diseases of the Lungs, No. 19.

† Diseases of the Blood-vessels, No. 100. 
matter, which extends into all the branches that are preserved. This was taken from a lady from whom, in the course of eleven years, Mr. Lawrence (to whom I am indebted for the history) removed at different times, both breasts and three large tumours which formed upon or between the cicatrices left after former operations. In the first breast removed (in May 1828) there appeared only an enormous enlargement of the mammary gland ; in the second (in 1834) a large tumour, resembling most the carcinoma hyalinum of Müller, was removed. In the three following operations performed in 1835,1837 and 1839 , the tumours removed were distinctly medullary. The patient died suddenly while she seemed in progress of recovery. In the examination after death (made by Mr. Crookes) the left lung was found everywhere adherent, congested, and extremely congested: the right lung was not adherent, but pale, œdematous and somewhat emphysematous : its vessels were healthy. There were no cancerous deposits in either lung, nor any in the bronchial glands. With the exception of the stomach, in which there were two or three small ulcers of the mucous membrane, all the abdominal and pelvic organs were healthy, and the abdominal walls and omentum were loaded with fat.

Fourthly-The branches of the pulmonary artery are often blocked up by clots formed during life in those who die with great œdema of the lungs. In some of these cases the coagulation is probably of the same kind as that which occurs in pulmonary apoplexy 
from disease of the left side of the heart, that is, it is consequent on the obstruction to the passage of blood through the capillaries. But in general some further condition appears to be necessary ; for old coagula are not found in all such cases, and it is probable that mere obstruction at the left side of the heart, if it were so complete as to permit the coagulation of large quantities of the blood during life, would prove fatal before the coagula could undergo any change, for it would affect almost equally the whole pulmonary circulation, and would produce a general and rapidly destructive stagnation. In one of the two following cases adduced as instances of coagula, thus occurring with œedema of the lungs, sufficient evidence of a morbid state of the blood is afforded by the signs of its early decomposition after death.

CASE II.

A man 60 years old, who had in early life drunk hard, and who had suffered during several successive winters from bronchitis and slight anasarca, supervening on emphysema of the lungs, was admitted into St. Bartholomew's Hospital in January 1841. He had his usual winter bronchitis, but it was accompanied by more than usual dropsy, the dyspnoea was greater than it had ever before been, and he was very weak and emaciated. After slowly sinking, he died in the middle of February.

At the examination eight hours after death, I found the following appearances:-The pericardium 
contained about an ounce of blood-stained fluid. The blood in the heart and large vessels was very softly clotted, and (as it is called) dissolved; and the walls of the vessels, and the fluids in contact with it, were deeply stained. Mixed with the soft clots, there was also a quantity of dirty-coloured fluid blood. All the cavities of the heart were rather larger than is natural ; and their walls were thin, soft, and weak. The borders of the tricuspid valve were slightly thickened and opaque. The pulmonary valves were healthy. The mitral valves had thickened and opaque free margins; their chordæ tendineæ were thick and opaque, but not shortened; the valves of the aorta were also, but in a slight degree, thickened. In the aorta itself there were a few small spots of morbid yellow deposit, and there were several of the same kind in the secondary branches of the pulmonary artery. There were almost universal adhesions of the pleuræ, and in those over the right lung, there was a plate of bone more than five inches square. In the only part where there were no adhesions, at the back of the right side of the chest, the pleural cavity contained some blood-stained fluid. The mucous membrane of the bronchi was rather turgid with blood, and many of them were filled by thick mucus. There was slight general emphysema of both lungs. Their texture was very œdematous and rotten, easily broken, and discharging a dirty greenish fluid, with but little air : at their lower parts they both had the 
appearance of compressed lungs; at the apex of the right, there was a group of old dry blackened tubercles. The branches of the pulmonary artery in nearly every part of both lungs, from those about $\frac{1}{6}$ th of an inch in diameter to the smallest that I could trace, were blocked up by round, firm, grumous, brittle clots. These, in many places, adhered closely to parts of the inner wall of the vessels; their colours were black, reddish, and white irregularly mixed, and in several places they appeared softened at their centres. Among the veins I examined the pulmonary, the azyga, superior and inferior cavæ, the porta (to its smaller branches), and the iliac veins, but all were healthy, and contained no coagula like those in the pulmonary arteries; but the femoral veins were filled by dark, firm, round coagula, with some of the blood discoloured; and these extended as far as the origins of the deep femoral veins, beyond which I did not dissect them.

The abdominal cavity contained about two pints of dark yellow fluid. The liver was small, pale, soft, and a little granular on its surface. The kidneys were small, pale, mottled, and slightly granular, and contained numerous small serous cysts. The digestive canal was healthy, except that its membranes were pale and soft; the spleen was soft, and pale purple. There was a stricture in the membranous part of the urethra, and the muscular coat of the bladder was hypertrophied. 
An habitually hard-drinking Irish-woman 50 years old, was received into St. Bartholomew's Hospital on the 23rd of February 1843, suffering with extreme dyspnœa, cough, dropsy, and albuminous urine. She could give no better account of herself, than that she had had these signs of disease in a less degree, "off and on," for five years. Her state was hopeless when she was admitted, and she died on the 27 th, after a long agony.

The body was examined 20 hours after death. It was generally anasarcous, and all the tissues were pale and flabby. The skull was thick and hard, there was a small tumour under the dura mater, and the brain was atrophied. The pericardium contained about four ounces of clear pale fluid; and there were some thick white spots upon the surface of the heart. The right auricle was very large, and its walls were thick and strong. The right ventricle also was greatly dilated and hypertrophied, and there were several small round and oval masses of bright yellow fibrine adhering in the recesses, between the fleshy columns at its apex; some of them were hollow. The left auricle and ventricle were dilated and hypertrophied, but less than those on the right side. All the muscular tissue of the heart was strong and robust. The tricuspid valve was thickened and opaque at its free edge, and appeared small in comparison with the orifice; the pulmonary valves and the trunk of the artery were large, 
but healthy in their structure. The mitral valve was contracted, thickened and opaque, but pliant ; there were several small vegetations on its margin, and the chordæ tendineæ were short and thick. The aortic valves were opaque, and slightly curled outwards ; there were small masses of firm fibrine, fixed on them, near the corpora Arantii. In the aortic arch there was abundant fatty deposit; and the lining membrane was thickened, opaque, white, and uneven. Each pleural cavity contained about a pint of clear fluid, with flakes of lymph on the right side. The mucous membrane of the trachea and bronchi was livid, and their cavities were full of thin frothy fluid. The upper parts of both lungs were excessively œdematous : the lower lobe of the right lung was soft and compressed, that of the left less compressed and congested, dark, and heavy, but not pneumonic. Many of the branches of the pulmonary arteries in all parts of both the lungs were blocked up by old coagula of partially discoloured blood. All the rest of the blood in the heart and large vessels was firmly but recently coagulated.

The abdominal cavity contained about three pints of serous fluid. The liver was of ordinary size, pale brown, anæmic, dry and soft. The spleen was large, dark, firm and adherent ; at the middle of its outer part there was a mass of soft bright yellow substance. The digestive canal was nearly healthy. The kidneys were small, nodulated and firm; their capsules were adherent; their surfaces mottled and granular, and their cortical substance thin. 
In the four classes of cases hitherto mentioned, the coagulation of the blood in the pulmonary arteries may be regarded as a secondary phenomenon; for it usually appears as the consequence, either chiefly or entirely, of the obstruction in the capillaries. Whenever that obstruction is complete, and prevails through the whole of both lungs, death by asphyxia must ensue before the arrested and coagulated blood can undergo any structural changes; and such changes can take place only when the capillary circulation can be carried on in some parts of the lungs, at the same time that in others the blood is stagnant.

There are, no doubt, some other diseases in which under the same essential conditions similar coagula are often formed. I have found them in a case of medullary tumour pressing upon the pulmonary veins ; in one of purulent deposits in the lungs coincident with phlebitis after amputation ;* and further examination will probably increase the number of diseases in which they will be acknowledged as important complications.

But there are other classes of cases in which the

* M. Craveilhier has figured a case of this kind connected with uterine phlebitis, (Anatomie Pathologique, Liv. XI. PI. II. III.) and similar cases have been observed by most of those who have written on phlebitis ; but, probably, there is a great difference between these cases, and those to which this paper refers, and in none of which any signs of inflammation of the coats of the pulmonary artery, or of acute phlebitis in any part of the body, have been found. 
formation of similar coagula appears to be a primary disease, or in which, at least, it cannot be regarded as the result of mere obstruction in the capillaries. Of these I have observed the three following examples :-

\section{CASE IV.}

A respectable married woman, 29 years old, and the mother of three children, was admitted into St. Bartholomew's Hospital under the care of Dr. George Burrows on the 5th of May 1843. She had had good health till the previous October, when she had rheumatism, which affected rather severely all the large joints in succession, and lasted for six weeks. After her recovery, she had occasional wandering pains, and three weeks before her admission, she noticed that her eyelids and face began to swell, and her joints, especially her knees and elbows, became swollen and painful, so that on the fourth day of her illness she was obliged to confine herself to bed. On her admission, the swelling of the joints had subsided, but they were stiff and tender, her feet and ancles were slightly œdematous; she was extremely weak and languid, complained of great thirst, and lay on her back, unable to lie on either side. She breathed 32 times in a minute, with some difficulty occasionally. Her pulse was 116 , and soft. Her complexion was sallow, with flushed cheeks, dark lines around the eyes, and sharpened features. The lips were dry and pale, the tongue dry, and furred, with a red tip. An 
abundant hot and acid-smelling perspiration was on the skin, and the urine was scantily but naturally secreted. Auscultation of the heart's sounds detected nothing unnatural.

In the three days after her admission no material change in her condition ensued. Nutritive diet and gentle purgatives and diaphoretics were given, and she appeared slightly improved ; but the great depression of the system, the anxiety of expression, and the rapid pulse and respiration, persisted. She perspired profusely, and was very thirsty. For two days the abdomen was distended, tympanitic and rather painful on pressure; on the next day she complained of great tenderness in the right iliac region; and on this day also (May 9th) the præcordial region was found tender, and on auscultation, a distant bellows-sound was heard at the base of the heart accompanying the systole. The state of the joints varied from day to day. On the 10th, the cheeks and forehead were covered with an erysipelatous blush, and numerous red acuminated papulæ had appeared upon the chest, the urine was very irritating, and smelt offensively, sloughs had begun to form upon the sacrum, and the weakness and depression were increased. On the morning of the 11 th, after passing a comfortable night, she was suddenly seized with a sensation of great tightness in the præcordial region, violent palpitation of the heart, and the most urgent dyspnœa. The attack lasted for an hour, and then she returned to nearly the same state as she had been in before it; but 
from this time she sank more rapidly. Upon auscultation, no respiratory murmur was heard below the right breast, and it was dull on percussion; the habitual dyspnœa became greater, the sloughs on the nates and sacrum extended, the abdomen became again tympanitic and tender, especially about the right iliac region, she vomited several times, and died in the afternoon of the 13th.

The history just read is taken from Dr. George Burrows's case-book, and he has added this note, which will serve to show what was the general character of the very anomalous symptoms which the case presented:- "The symptoms were very obscure at the time of the patient's admission. I regarded the case as one of rheumatism occurring in a woman of feeble constitution. Upon the appearance of the remarkable symptoms on the morning of the 1lth of May I formed the opinion that she was attacked with passive pneumonia of the right lung, and I considered it highly probable that the constitution was suffering under the depressing effects of the typhus-poison, for there were several severe cases of typhus fever in the beds adjacent to that of the patient at this time."

On the examination 40 hours after death, I found all the blood which appeared in the ordinary course of the dissection either fluid or coagulated in soft black masses. The pericardium contained half an ounce of fluid; there were a few slender adhesions between the trunks of the large vessels, and on the surface of the heart were two white spots. The 
heart was of ordinary size, and its parts well-proportioned; but its tissue was rather weak and pale. The valves on its right side were thin, clear and healthy; those on the left side were a little thickened and opaque, but quite pliant. In the coats of the aorta were a few scattered fatty deposits; those of the pulmonary artery were healthy. Each pleural sac contained a small quantity of clear fluid, and at the apex of each lung there was an adhesion with a few dry and shrivelled tubercles beneath it. The lower lobes of both lungs were slightly but generally oedematous, and gorged with blood. The upper lobes, with the exception of the small groups of old tubercles, were healthy. The trachea and bronchi were also healthy, and contained a small quantity of mucus. Nearly half the branches of the pulmonary artery, from those of the second order, to those of the fifth and sixth (and probably to yet smaller branches), were blocked up by old coagula of blood. These were cylindrical, soft and grumous, and in colour were a mixture of pale pink and dirty greyish white, with spots and blotches of deep crimson. They were not more numerous in one lung than in the other, and were irregularly scattered through all parts of each. They did not quite fill the vessels which contained them, but at various parts they adhered closely to the walls. The trunk of the pulmonary artery, and many of the branches which did not contain coagula of the kind just described, contained fluid and softly clotted black blood. The vOL. XXVII. 
abdominal and pelvic organs were healthy in their structure, but very pale, and weak.

\section{CASE V.}

A widow 70 years old, thin and emaciated, was admitted under Dr. George Burrows on the 28th of December 1843. She had always had good health, with the exception of a slight cough during the last three winters. Five weeks before her admission she was attacked by a bad cold, with some cough, but no pain in the chest, or dyspnœa. A fortnight after this, her legs began to swell. When admitted, she had a sallow countenance, with livid lips, a very irregular and feeble pulse, beating nearly 100 times in a minute, and was breathing laboriously 32 times in a minute. She had no pain, but a slight degree of tightness in the chest, and expectorated a little frothy tenacious fluid. The lower extremities were odematous, and there were some small abrasions, with apparent sloughing, of the cuticle of the legs. On the day after her admission, she seemed to improve a little; but next day she sank rapidly, and Dr. Burrows " remarked an extreme hurry of the circulation, with feebleness of the pulse and great prostration of strength, very similar to those observed in the preceding case."

In the examination 36 hours after death, I found the lungs of ordinary size, but deeply wrinkled, when collapsed. There were a few scattered adhesions on their anterior surfaces. Their tissue was 
generally light and crepitant, and in a few parts slightly œdematous; the air-cells were of ordinary size, except about the lower and anterior margins of the lobes, where there was a moderate degree of emphysema. In a few parts the pulmonary tissue was compressed; and at the anterior and lower border of the left lung was a small patch of compact pulmonary apoplexy, with some diffused apoplexy around it. The bronchi were of ordinary size ; their mucous membrane thin, pale, and firm; their tubes full of thick semi-transparent viscid mucus. In each lung, one of the superior and one of the inferior main branches of the pulmonary artery were blocked up by a large, firm, mottled, clot of blood, which, from itself as a trunk, sent branches into two or more of the next order of branches of the artery. The colours of the clots were black, deepcrimson, rusty, pink and yellowish, in various irregularly mingled shades; they were moderately firm, of nearly uniform consistence throughout, and capable of being rubbed into a thick grumous substance; they adhered so firmly by parts of their surfaces to the adjacent walls of the vessels, that they could not be smoothly removed. The branches of the largest clots did not extend far into the arteries, but terminated abruptly in the arterial ramifications immediately proceeding from those in which they lay. But beyond their terminations, many smaller branches of the pulmonary arteries in all parts of the lungs contained short, firm, dry, mottled, and adherent clots, variously coloured. None of these 
was long or much ramified; none was continued through more than two branches; and many of them were not more than half or three-quarters of an inch long; some were even shorter, and lay like bits of larger clots upon the walls of the vessels. They had all the same characters as those already described; and the portions of lung adjacent to and beyond them, were not different from the rest.

Moreover, in several of the larger branches of the pulmonary artery, there were appearances of clots of blood formed like those just described, and having been still further altered and organized. These were pale, semi-transparent, soft, and flattened narrow bands, attached firmly to the walls of the artery, and presenting all the characters of the organized clots which I have sometimes seen adhering to the walls of divided arteries. They were from one quarter of an inch to an inch in length, and about one-tenth of an inch wide; a few were fixed, in their whole length, to the walls of the artery, but most of them by their ends only, so that a probe could be passed under them. Among the clots there were all gradations, between those last and those first described; and in one instance, one of the largest of the more recent clots was continuous, with a flat, semi-transparent, and adherent portion, like those which had existed for the longest time. (See Plate III. fig. 3.)

The branches of the pulmonary arteries which were thus obstructed, were smooth and polished internally, but in many places had fine, scattered, 
and grouped grains of yellow deposit in their coats. They were all of natural size; and the pulmonary veins were healthy.

The cavities of the right side of the heart were considerably dilated and hypertrophied. Both of them were distended by soft and uniformly black coagulum, slightly adhering to their walls; and in the appendix of the right auricle the coagulum had become firm, mottled, and closely adherent, somewhat like those in the pulmonary arteries. The left auricle and ventricle were also dilated and hypertrophied, but in a less degree than the right; they contained a smaller quantity of similar soft black coagulum. The tricuspid valve was healthy; the pulmonary valves were large, and in small patches, opaque, but thin and pliant; the mitral valve was slightly thickened, and had several spots of soft yellow substance beneath its ventricular surface ; the aortic valves were also irregularly thickened and opaque, with enlarged corpora Arantii, bearing small warty growths; but both they and the mitral valves might be deemed able to discharge their functions. The aorta was large, with numerous yellow deposits in its coats.

The digestive canal was healthy. The liver was small, tough, contracted, and granulated, and a great part of its surface was covered by long adhesions. The gall-bladder was full of calculi. The kidneys were small and somewhat granulated, with adherent capsules, and numerous cysts full of transparent fluid. The uterus prolapsed beyond the 
labia. The large veins and the systemic arteries, as far as they were examined, were healthy.

CASE VI.

A girl, twenty years old, who had long lived in the miseries of poverty and prostitution, was admitted under Mr. Stanley, for gonorrhœa, about seven weeks before her death. She was pale, haggard, and scarcely able to walk. She had a slight cough, without expectoration, occasional anasarca of the lower extremities, and violent palpitation, with increased impulse of the heart. She had been subject to sudden fainting fits. In bed, she lay constantly on her back, as if in a state of great exhaustion, but she did not appear to have difficulty in breathing. Her pulse was rapid and feeble. A loud bellows-murmur accompanied and terminated the first sound of the heart; it was heard over the whole chest, but most distinctly at the base of the heart, and in a line extending from the middle of the base upwards, and towards the left shoulder. The same sound was heard also behind the chest, as well as in front. She had not menstruated regularly for four months; her urine was alkaline and albuminous; and on her admission she had some ascites and cedema of the legs. During the first month of her stay in the hospital, her condition appeared slowly improving; but then effusion into the chest, and signs of pleuro-pneumonia came on, and she soon died.

The pericardium contained about three ounces of 
clear fluid. The blood in the heart and large vessels was firmly coagulated and separated in dark clots with superjacent buffy coats of transparent fibrine. The right auricle was somewhat dilated and hypertrophied; the right ventricle was so in a much greater degree, and there were several deposits of a firm, pale, yellow substance upon, and beneath its lining membrane, near the orifice of the pulmonary artery. The left auricle and ventricle were healthy. The tricuspid valve was healthy. The pulmonary valves were only two in number; they were thickened and opaque, and had on their free borders and adjacent surfaces large growths of brownish-yellow, soft, fibrine-like substance, which firmly adhered to them, and which had in their interior small grains of earthy substance. The largest of these growths or deposits was of an irregularly triangular form, having its base attached to the largest and posterior of the valves, and its.apex reaching to the opposite wall of the artery. Behind the valves, and on the lining membrane of the trunk and division of the pulmonary artery, there were seven or eight warty growths, from two to four lines broad and a line and a half high, composed of a pale yellow and nearly transparent substance, like that of the deposits within the ventricle, but firmer and more firmly adherent. Behind the largest of the valves there was a distinct ulcer of the lining membrane of the artery, nearly half an inch in diameter, with a rough uneven base and shreddy margins; and a smaller ulcer of the same kind was situated at the angle of bifurcation of the artery. The mitral and 
aortic valves were healthy, and the aorta was slightly yellow-spotted.

The right pleura had extensive adhesions; the remains of its cavity contained six ounces of clear yellow fluid. The left pleura contained a pint of serous fluid with flakes of lymph, and had an abundance of recent vascular lymph upon its surfaces. The trachea and bronchi were healthy and contained some tough mucus. In both lungs the upper lobes were pale, dry, light, and slightly emphysematous. In the lower lobes of both, all the pulmonary arteries, from the origin of the main branch to the most distant branches that I traced, were full of old fibrinous coagula, which branched in correspondence with the vessels in which they lay. The largest parts of the coagula were pale yellow, dry, very firm, laminated and closely adherent to nearly the whole circumference of the arterial walls, which when cleared were found dark, rough, and thin. The smaller branches of the coagula had exactly the same characters, except that they were darker. In the lower lobes of the lungs there were also about twenty firm and compact masses of pulmonary apoplexy of very irregular form, and measuring from half-an-inch to two inches in diameter. The circumferences of them all' were of a deep and dark crimson colour ; and of those which were just beneath the pleura the external surfaces had the same ordinary colour of pulmonary apoplexy. But in their interiors the blood was in various degrees decolorized, and their sections presented different shades of a pale greyish-yellow 
colour. The parts of the lungs adjacent to them were rather firmer than the rest, and odematous; the intervening parts were healthy.

The abdomen contained about a pint of yellow serum, with long strings of soft lymph. The liver was pale; but otherwise both it and the gall-bladder were healthy. The spleen was large, firm, and adherent to the abdominal walls; it was dark, and there were, both in it and on its surface, many still darker spots, like ecchymoses. The renal capsules were large, firm, and fleshy. On the mucous membrane of the stomach there were traces of three or four small superficial cicatrices; but in other respects it was healthy. In the ileum there were several apparently old, broad, transverse bands of irregular ulceration, covered by uneven lymph, but vascular. The kidneys were healthy in texture, but pale. There were several superficial ulcers in the upper part of the vagina.

There is so little similarity in the symptoms presented by these three patients, that I cannot draw any certain deduction concerning the real nature of their diseases. I will therefore only offer these few observations on them.

In none of the cases (except the last) is there any indication that the coagulation of the blood was the consequence of disease of the pulmonary arteries. In some of them, fine grains of yellow fatty deposit lay beneath the anterior lining membrane; and I will take this occasion to state, that it is erroneous to suppose that this disease is very rare in the 
pulmonary arteries. It is very rarely seen in the trunk of the artery ; but in the branches of the second, third, and further orders, it occurs to some extent in half the persons who die beyond the middle age. It is not always associated with a greater degree of the same disease in the aortic system; but it rarely attains to a state materially affecting the functions of the artery, and is rarely complicated by the deposition of earthy matter. However, there is no reason to believe that any connection exists between the coagulation of the blood in the cavities of the arteries and this disease in their coats.

The clots in the last case were distinguished by being laminated, as if formed by the deposition of successive layers from the wall towards the axis of the artery. In all the others, the particles of the clots were irregularly arranged; they were throughout various in both colour and consistence. Some were partially softened; indicating, I suppose, that the blood had died before the general death of the body; others, in which the blood had, probably, become further organized, had acquired firm adhesions to the walls of the vessels; and in the fifth case, it was evident that the clots were fully organized, and had acquired organic connections with the adjacent parts. In this respect the case is pecu- liarly interesting, for it proves that blood, though coagulated in circumstances of disease, is yet capable of further organization, when placed in conditions compatible with the maintenance of its life; and further, that the coagulation of the blood in the 
large pulmonary arteries, is not necessarily either the cause or the attendant of fatal disease; for several weeks must have been occupied in effecting the complete organization to which these clots had attained. However, it must be remarked as a peculiar character of this case, that the clots were not generally long and branching, but were in small bits, many of which occupied only a part of the calibre of the artery.

The last case related, is made the more remarkable by the occurrence of so rare an affection as disease of the valves and trunk of the pulmonary artery. In its general characters, it much resembles the cases related by Dr. Bright,* of obstructions of the veins by fibrinous coagula formed during life, in females debilitated from various circumstances. There is not, indeed, any mention of coagula in the pulmonary arteries in those cases; nor were there any coagula in the veins in that which $I$ have related. Yet they are all, in many respects, similar; and one of the observations published by M. Baron, $\dagger$ supplies the very fact which is necessary to establish the connection between them; for in it the circumstances during life were very like those observed in these cases, and after death old fibrinous coagula were found in both the veins and the pulmonary artery.

In conclusion, though it is not connected with

* Reports of Medical Cases, vol. ii., p. 63.

† L. c. p. 18. 
the diseases to which this paper especially refers, I would point to the fact of there being only two valves in the pulmonary artery, in the case last related. It has never, I believe, been noticed (though there need be no doubt about the fact), that in the majority of cases in which only two valves have been found in the aorta or pulmonary artery, those valves have been diseased, and often extremely diseased. This fact is very important; for it affords evidence (which is confirmed by several other observations), that parts which, from defective or erroneous development, are wrongly shaped, are often, at the same time, imperfect in their tissue, and are therefore very liable to disease; and it is thus, moreover, a striking example of those congenital defects of the composition of the textures, which, though they are little studied, are much more common and important than the more obvious congenital defects of shape. 

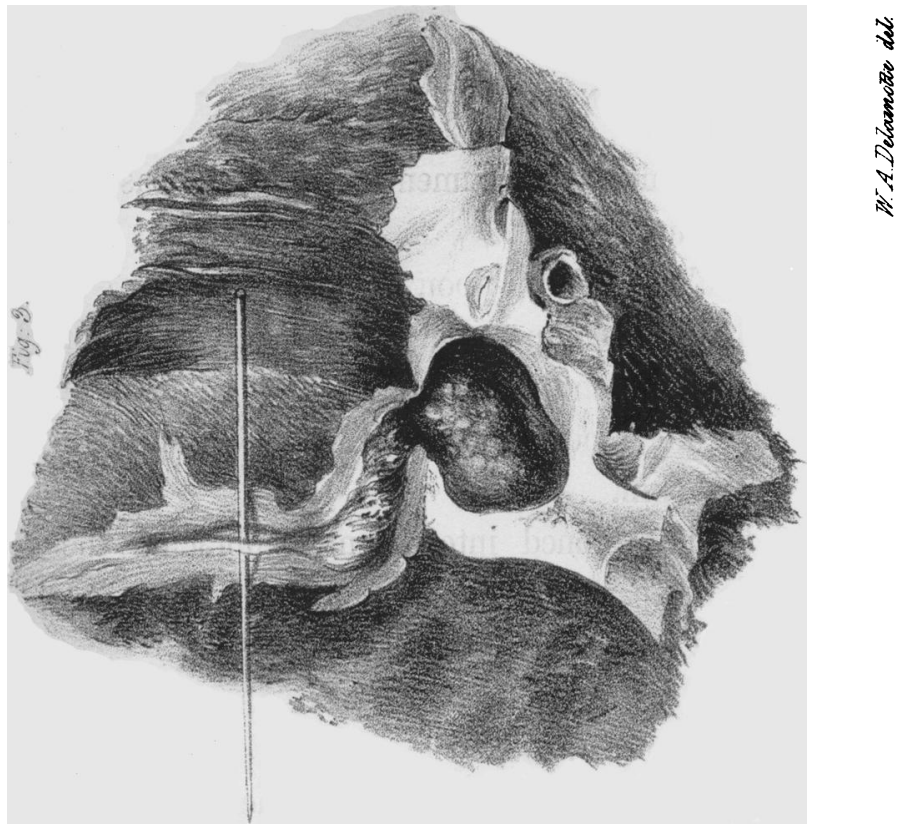

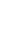

3

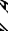
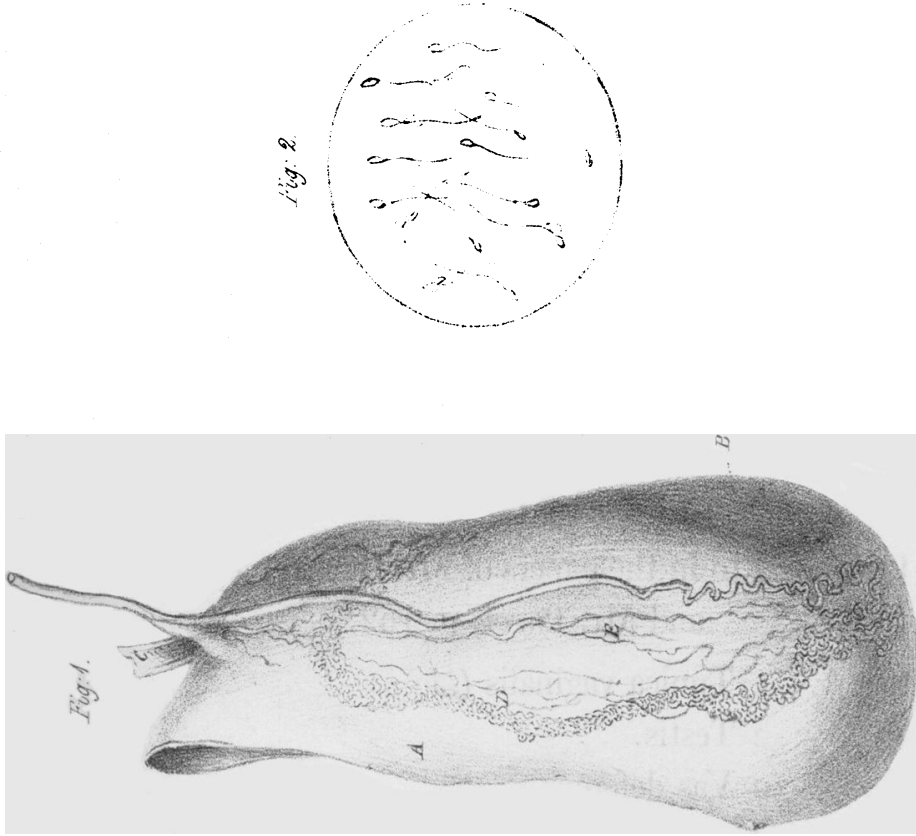

3.

ș

5

ำ 